Case Report

\title{
Anaerobic Spondylodiscitis due to Fusobacterium Species: A Case Report Review of the Literature
}

\author{
Tiffany N. Latta, ${ }^{1}$ Aimee L. Mandapat, ${ }^{2,3}$ and Joseph P. Myers ${ }^{2,3}$ \\ ${ }^{1}$ Department of Medicine, Summa Akron City Hospital, Akron, OH 44304, USA \\ ${ }^{2}$ Division of Infectious Diseases, Department of Medicine, Summa Akron City Hospital, Akron, OH 44304, USA \\ ${ }^{3}$ Section of Infectious Diseases, Department of Internal Medicine, Northeast Ohio Medical University, Rootstown, OH 44272, USA
}

Correspondence should be addressed to Joseph P. Myers; joseph.myers@summahealth.org

Received 11 February 2015; Accepted 16 April 2015

Academic Editor: Alexandre R. Marra

Copyright (C) 2015 Tiffany N. Latta et al. This is an open access article distributed under the Creative Commons Attribution License, which permits unrestricted use, distribution, and reproduction in any medium, provided the original work is properly cited.

\begin{abstract}
Spondylodiscitis caused by Fusobacterium species is rare. Most cases of spontaneous spondylodiscitis are caused by Staphylococcus aureus and most postoperative cases are caused by Staphylococcus aureus or coagulase-negative staphylococci. Escherichia coli is the most common Gram-negative organism causing spondylodiscitis. Fusobacterium species are unusual causes for anaerobic spondylodiscitis. We report the case of a patient with spontaneous L2-L3 spondylodiscitis, vertebral osteomyelitis, and epidural abscess caused by Fusobacterium species and review the literature for patients with Fusobacterium spondylodiscitis.
\end{abstract}

\section{Introduction}

Spondylodiscitis due to Fusobacterium species is unusual with only 15 cases having been previously reported [113]. Hematogenous spondylodiscitis is most often caused by Staphylococcus aureus [14-16]. Postoperative spondylodiscitis is most commonly due to staphylococcal species [17]. The most common etiologic agent of spontaneous Gram-negative spondylodiscitis is Escherichia coli [18]. Propionibacterium and Bacteroides species are the most commonly reported causes for anaerobic spondylodiscitis [19]. We report a patient with spontaneous L2-L3 spondylodiscitis due to Fusobacterium species and review the literature for other reports of this disease.

\section{Materials and Methods}

PubMed and Google Scholar searches were performed using the words discitis, diskitis, spondylodiscitis, vertebral osteomyelitis, and Fusobacterium looking for previously reported cases of discitis and osteomyelitis caused by Fusobacterium species. References from each of the articles obtained by these searches were also reviewed for further pertinent case reports.

\section{Case Report}

A 57-year-old man with a past history of asthma was admitted to the hospital with a three-month history of gradually worsening low back pain aggravated by movement and not improved with rest, cyclobenzaprine, or a tapering course of prednisone. Physical examination revealed multiple abscessed teeth and tenderness to palpation over the second and third lumbar vertebrae. The neurologic examination was normal. Complete blood count revealed a WBC of $13,600 / \mathrm{cmm}$ (normal: $4.400-11,300 / \mathrm{cmm}$ ) with $93.4 \%$ granulocytes, $5.6 \%$ lymphocytes, and $1.0 \%$ monocytes. Erythrocyte sedimentation rate was $6 \mathrm{~mm} / \mathrm{hr}$ (normal: $0-10 \mathrm{~mm} / \mathrm{hr}$ ). Creactive protein was $3.68 \mathrm{mg} / \mathrm{dL}$ (normal: $<0.6 \mathrm{mg} / \mathrm{dL}$ ). MRI of the lumbar spine revealed severe L2-L3 discitis, contiguous L2 and L3 vertebral osteomyelitis with end-plate erosion, and epidural and psoas muscle abscesses. CT-guided biopsy was performed and a pure culture of Fusobacterium species was isolated from anaerobic cultures of the disc-space tissue aspirate. Fungal and mycobacterial cultures were negative. The abscessed teeth were extracted and the patient was successfully treated with ertapenem, one gram intravenously daily for 8 weeks. Follow-up examination 6 months later showed mild residual pain and a normal physical examination. 


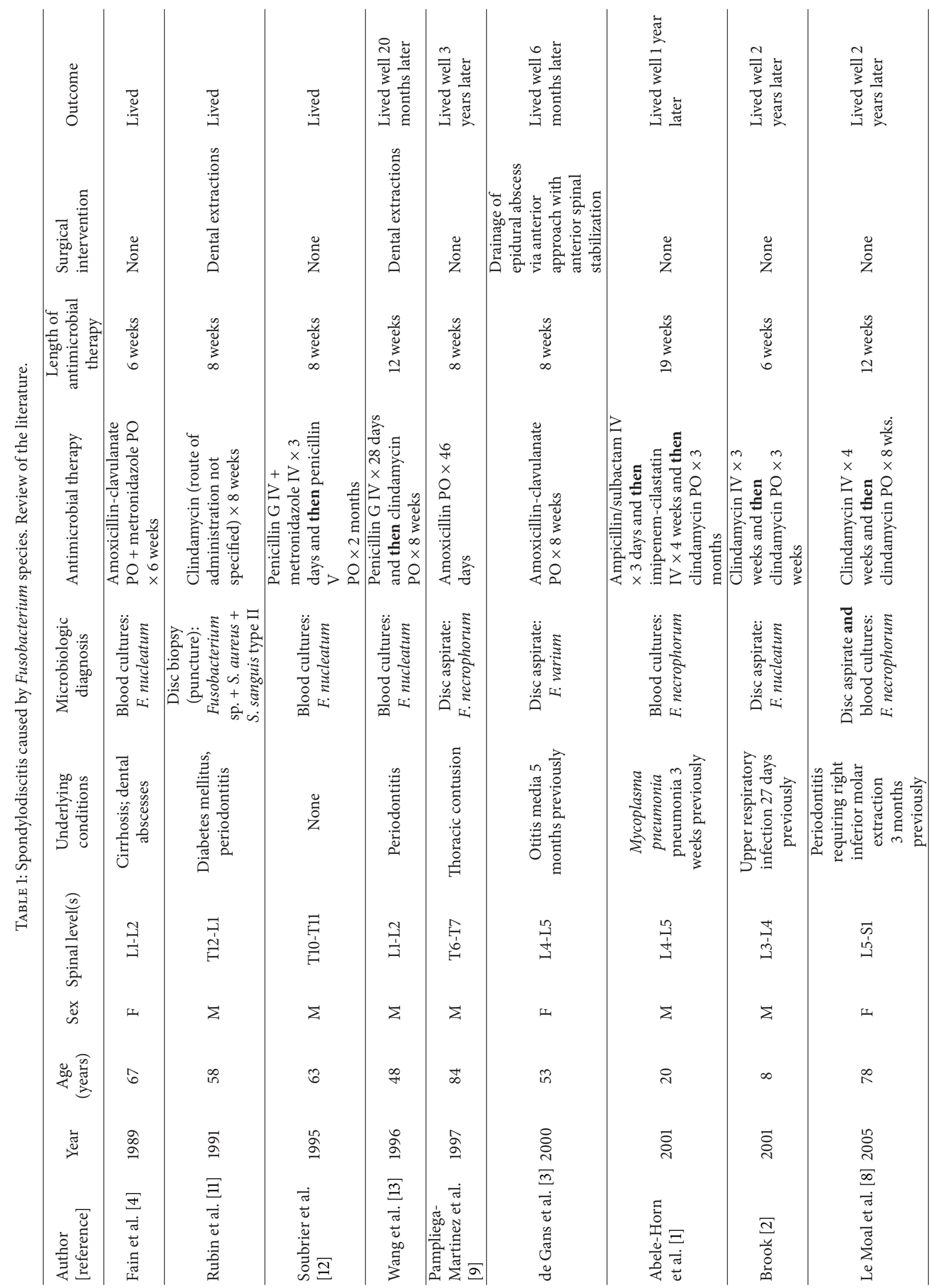




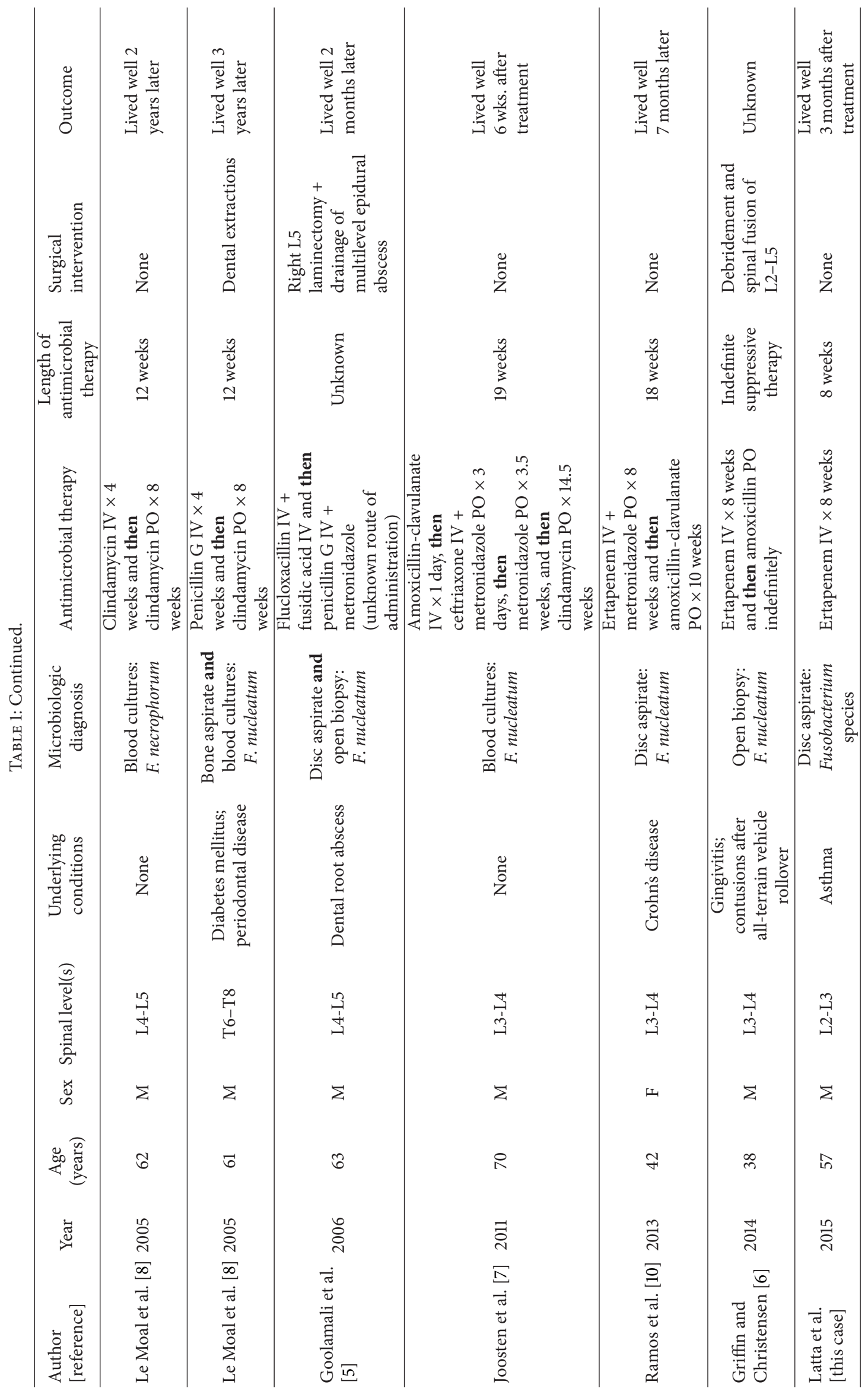




\section{Discussion}

Fusobacterium is a genus of obligately anaerobic filamentous Gram-negative rods that are members of the phylum Fusobacteria [20, 21]. They can be divided into 13 species that inhabit the oral, gastrointestinal, upper respiratory tract, and vaginal mucosa [22]. Fusobacterium species can be variable in Gram stain morphology and display a range of cellular morphologies from coccoid, pleomorphic spherules to rod shaped organisms. Rods can be short with rounded ends or long with pointed ends. Most Fusobacterium species are indole positive and produce butyric acid during the fermentation of glucose $[20,21]$. Fusobacterium nucleatum is the species most commonly described causing human infection and Lemierre's syndrome caused by Fusobacterium necrophorum is the best known infection associated with the Fusobacteria $[23,24]$. Fusobacterial infections of almost every anatomic site have been reported $[23,24]$. Bacteremia caused by Fusobacterium species is unusual but not rare [25-27]. Several patients with fusobacterial vertebral and paravertebral infections without classic spondylodiscitis have also been reported [28-31].

Patients with fusobacterial spondylodiscitis reported in the literature are listed in Table 1 . Patient ages ranged from 8 to 84 years with a mean of 54.5 years. There were 12 men (75\%) and 4 women (25\%). The most common spinal level for spondylodiscitis was the lumbar area. There were 4 patients with thoracic disease (25\%) and 12 with lumbar disease (75\%). L3-L4 and L4-L5 discitis were most common with 4 patients with disease at each of those sites. One patient had multilevel disease (T6-T8). Three patients had no underlying illness. Seven of 16 patients (43.75\%) had significant oral pathology: 5 with periodontitis and 2 with dental root abscess. Fusobacterium spondylodiscitis was microbiologically confirmed by blood culture alone in 6 cases (37.5\%), by tissue biopsy culture in 8 cases $(50.0 \%)$, and by blood and tissue biopsy cultures in 2 cases (12.5\%). The duration of antimicrobial therapy was unknown in 2 patients and ranged from 6 to 19 weeks in the other 14 patients with an average of 11.1 weeks. Treatment regimens included penicillin, metronidazole, clindamycin, carbapenem, or some combination of these agents. Details of treatment are presented in Table 1 . All 15 patients for whom information was available survived. Three patients required surgical intervention: one had drainage of an epidural abscess and anterior spinal stabilization; one had urgent laminectomy and drainage of an epidural abscess; and one had disc-space debridement and a spinal fusion.

Fusobacterium is an unusual cause of infectious spondylodiscitis but responds well to medical/surgical therapy with no deaths recorded in the reported patients. Blood, disc tissue, or psoas abscess cultures in our patients often required 72 hours of incubation before there was sufficient growth to identify a pathogen. Thus, any patient suspected of having culture-negative spondylodiscitis should have anaerobic cultures performed with an adequate time allowed for incubation of biopsy specimens before one proceeds with a detailed work-up for other etiologies of culture-negative disease. Treatment with at least 6 weeks of antimicrobial therapy was curative in all reported patients, although three patients required surgical intervention for complications such as paraspinal phlegmon. Fusobacterium isolates are usually sensitive to penicillin, ampicillin, amoxicillin/clavulanate, piperacillin/tazobactam, clindamycin, metronidazole, moxifloxacin, tigecycline, imipenem, and ertapenem [20, 21]. Because Fusobacterium spp. are often part of polymicrobial infectious flora, therapy should be guided by both available sensitivity testing and the potential presence of other aerobic/anaerobic organisms in cultures from the site of infection. Because of its once daily administration schedule and its antimicrobial spectrum, ertapenem is an especially attractive treatment option when outpatient parenteral antimicrobial therapy (OPAT) is being used. Moxifloxacin, a fluoroquinolone with high oral bioavailability, once daily administration, and aerobic/anaerobic spectrum, is an attractive oral alternative to OPAT with its requisite peripherally inserted central venous catheter [20,21].

\section{Conflict of Interests}

The authors declare that there is no conflict of interests regarding the publication of this paper.

\section{References}

[1] M. Abele-Horn, P. Emmerling, and J. F. E. Mann, "Lemierre's syndrome with spondylitis and pulmonary and gluteal abscesses associated with Mycoplasma pneumoniae pneumonia," European Journal of Clinical Microbiology and Infectious Diseases, vol. 20, no. 4, pp. 263-266, 2001.

[2] I. Brook, "Two cases of diskitis attributable to anaerobic bacteria in children," Pediatrics, vol. 107, no. 2, article e26, 2001.

[3] J. de Gans, S. Schade van Westrum, E. Kuijper, and G. R. Schaap, "Earache and back pain," The Lancet, vol. 355, no. 9202, p. 464, 2000.

[4] O. Fain, T. Bardin, I. Cheour, F. Legmann, A. Felten, and D. Kuntz, "Spondylodiscitis caused by Fusobacterium nucleatum. Apropos of 1 case," Revue du Rhumatisme et des Maladies OsteoArticulaires, vol. 56, no. 4, pp. 339-340, 1989.

[5] S. I. Goolamali, M. T. Carulli, and U. M. Davies, "Spinal abscess and mitral valve endocarditis secondary to asymptomatic fusobacterium-induced dental abscess," Journal of the Royal Society of Medicine, vol. 99, no. 7, pp. 368-369, 2006.

[6] A. T. Griffin and D. Christensen, "Fusobacterium spondylodiscitis: case report and literature review," Diagnostic Microbiology and Infectious Disease, vol. 78, no. 4, pp. 491-493, 2014.

[7] A. Joosten, J. Verhaegen, and E. Van Wijngaerden, "Fusobacterium nucleatum spondylodiscitis: case report and literature review," Clinical Microbiology Newsletter, vol. 33, no. 15, pp. 115117, 2011.

[8] G. Le Moal, L. Juhel, G. Grollier, C. Godet, I. Azais, and A. Roblot, "Vertebral osteomyelitis due to Fusobacterium species: report of three cases and review of the literature," Journal of Infection, vol. 51, no. 2, pp. E5-E9, 2005.

[9] T. Pampliega-Martinez and A. Arenas-Planelles, "Espondilodiscitis por Fusobacterium necrophorum: a propósito de un caso," Revista Española de Cirugía Osteoarticular, vol. 32, no. 192, pp. 284-288, 1997.

[10] A. Ramos, E. Berbari, and P. Huddleston, "Diagnosis and treatment of fusobacterium nucleatum discitis and vertebral 
osteomyelitis: case report and review of the literature," Spine, vol. 38, no. 2, pp. E120-E122, 2013.

[11] M. M. Rubin, R. J. Sanfilippo, and R. S. Sadoff, "Vertebral osteomyelitis secondary to an oral infection," Journal of Oral and Maxillofacial Surgery, vol. 49, no. 8, pp. 897-900, 1991.

[12] M. Soubrier, Z. Urosevic, J. J. Dubost, J. M. Ristori, and J. L. Bussière, "Spondylodiscitis caused by Fusobacterium nucleatum. Apropos of a case," Presse MEédicale, vol. 24, no. 21, pp. 989-991, 1995.

[13] T.-D. Wang, Y.-C. Chen, and P.-J. Huang, "Recurrent vertebral osteomyelitis and psoas abscess caused by Streptococcus constellatus and Fusobacterium nucleatum in a patient with atrial septal defect and an occult dental infection," Scandinavian Journal of Infectious Diseases, vol. 28, no. 3, pp. 309-310, 1996.

[14] L. Cottle and T. Riordan, "Infectious spondylodiscitis," Journal of Infection, vol. 56, no. 6, pp. 401-412, 2008.

[15] T. Gouliouris, S. H. Aliyu, and N. M. Brown, "Spondylodiscitis: update on diagnosis and management," Journal of Antimicrobial Chemotherapy, vol. 65, supplement 3, pp. iiill-iii24, 2010.

[16] J. M. Nolla, J. Ariza, C. Gómez-Vaquero et al., "Spontaneous pyogenic vertebral osteomyelitis in nondrug users," Seminars in Arthritis and Rheumatism, vol. 31, no. 4, pp. 271-278, 2002.

[17] M. E. Jimenez-Mejias, J. de Dios Colmenero, F. J. Sánchez-Lora et al., "Postoperative spondylodiskitis: etiology, clinical findings, prognosis, and comparison with nonoperative pyogenic spondylodiskitis," Clinical Infectious Diseases, vol. 29, no. 2, pp. 339-345, 1999.

[18] K.-H. Park, O. H. Cho, M. Jung et al., "Clinical characteristics and outcomes of hematogenous vertebral osteomyelitis caused by gram-negative bacteria," Journal of Infection, vol. 69, no. 1, pp. 42-50, 2014.

[19] M. U. Saeed, P. Mariani, C. Martin et al., "Anaerobic spondylodiscitis: case series and systematic review," Southern Medical Journal, vol. 98, no. 2, pp. 144-148, 2005.

[20] W. S. Garrett and A. B. Onderdonk, "Bacteroides, Prevotella, Porphyromonas, and Fusobacterium species (and other medically important anaerobic Gram-negative bacilli)," in Mandell, Douglas, and Bennett's Principles and Practice of Infectious Diseases, J. E. Bennett, R. Dolin, and M. J. Blaser, Eds., pp. 27732780, WB Saunders, Philadelphia, Pa, USA, 2015.

[21] E. Kononen, W. G. Wade, and D. M. Citron, "Bacteroides, Porphyromonas, Prevotella, Fusobacterium, and other anaerobic gram-negative rods," in Manual of Clinical Microbiology, J. Versalovic, Ed., pp. 858-880, ASM Press, Washington, DC, USA, 10th edition, 2011.

[22] D. M. Citron, "Update on the taxonomy and clinical aspects of the genus Fusobacterium," Clinical Infectious Diseases, vol. 35, no. 1, pp. S22-S27, 2002.

[23] P. J. Huggan and D. R. Murdoch, "Fusobacterial infections: clinical spectrum and incidence of invasive disease," Journal of Infection, vol. 57, no. 4, pp. 283-289, 2008.

[24] E. Pett, K. Saeed, and M. Dryden, "Fusobacterium species infections: clinical spectrum and outcomes at a district general hospital," Infection, vol. 42, no. 2, pp. 363-370, 2014.

[25] K. Afra, K. Laupland, J. Leal, T. Lloyd, and D. Gregson, "Incidence, risk factors, and outcomes of Fusobacterium species bacteremia," BMC Infectious Diseases, vol. 13, article 264, 2013.

[26] A.-M. Bourgault, F. Lamothe, P. Dolcé, L. Saint-Jean, and P. Saint-Antoine, "Fusobacterium bacteremia: clinical experience with 40 cases," Clinical Infectious Diseases, vol. 25, no. 2, pp. S181-S183, 1997.
[27] E. A. Goldberg, T. Venkat-Ramani, M. Hewit, and H. F. Bonilla, "Epidemiology and clinical outcomes of patients with Fusobacterium bacteraemia," Epidemiology and Infection, vol. 141, no. 2, pp. 325-329, 2013.

[28] H. L. Freedman and W. F. Cashman, "Vertebral osteomyelitis caused by Fusobacterium nucleatum: a case report," Orthopedics, vol. 2, no. 4, pp. 366-369, 1979.

[29] D. H. Kempen, M. van Dijk, A. I. Hoepelman, F. C. Oner, and J. J. Verlaan, "Extensive thoracolumbosacral vertebral osteomyelitis after Lemierre syndrome," European Spine Journal, vol. 23, no. 9, 2014.

[30] B. Peer Mohamed and L. Carr, "Neurological complications in two children with Lemierre syndrome," Developmental Medicine and Child Neurology, vol. 52, no. 8, pp. 779-781, 2010.

[31] J. L. Sanmillán, I. Pelegrín, D. Rodríguez, C. Ardanuy, and C. Cabellos, "Primary lumbar epidural abscess without spondylodiscitis caused by Fusobacterium necrophorum diagnosed by 16S rRNA PCR," Anaerobe, vol. 23, pp. 45-47, 2013. 


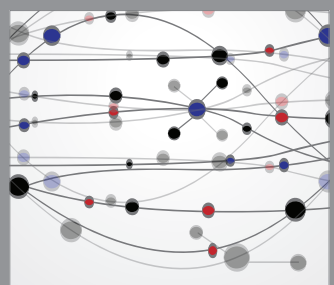

The Scientific World Journal
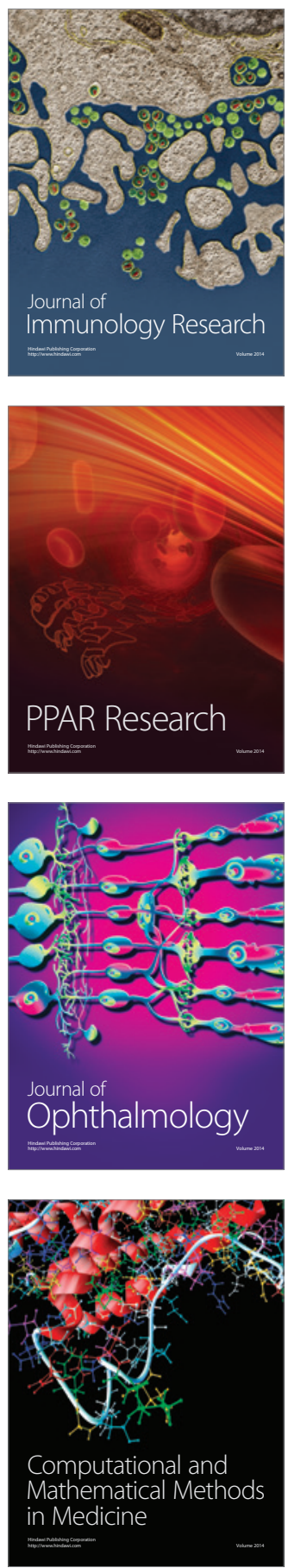

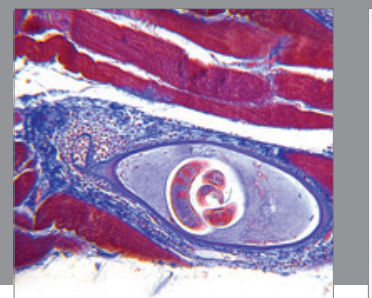

Gastroenterology

Research and Practice
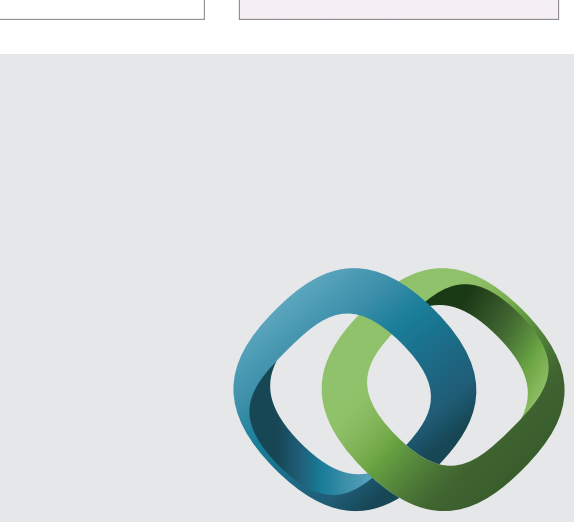

\section{Hindawi}

Submit your manuscripts at

http://www.hindawi.com
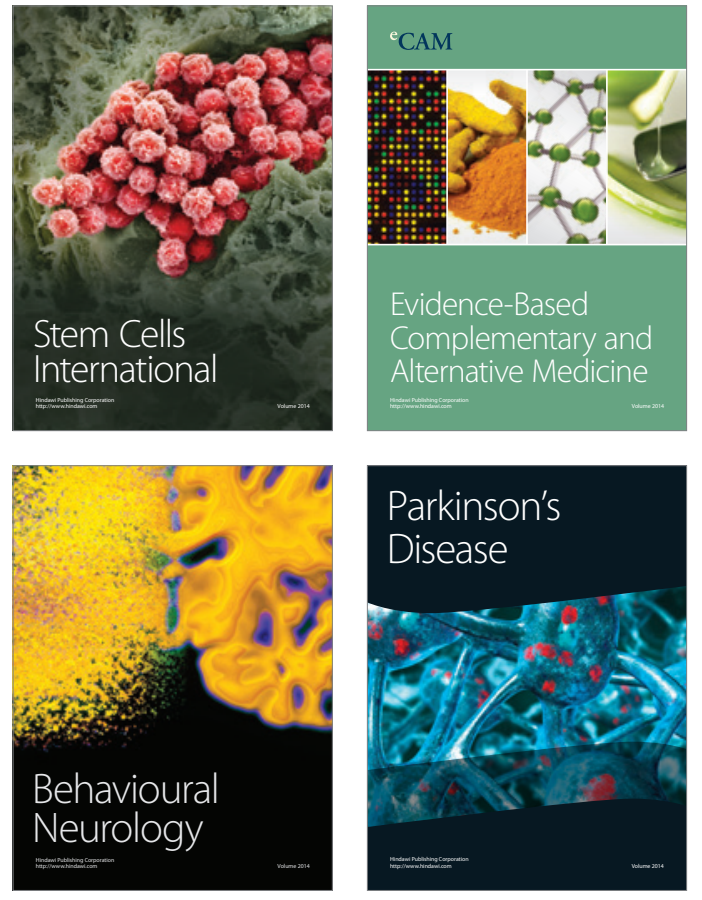
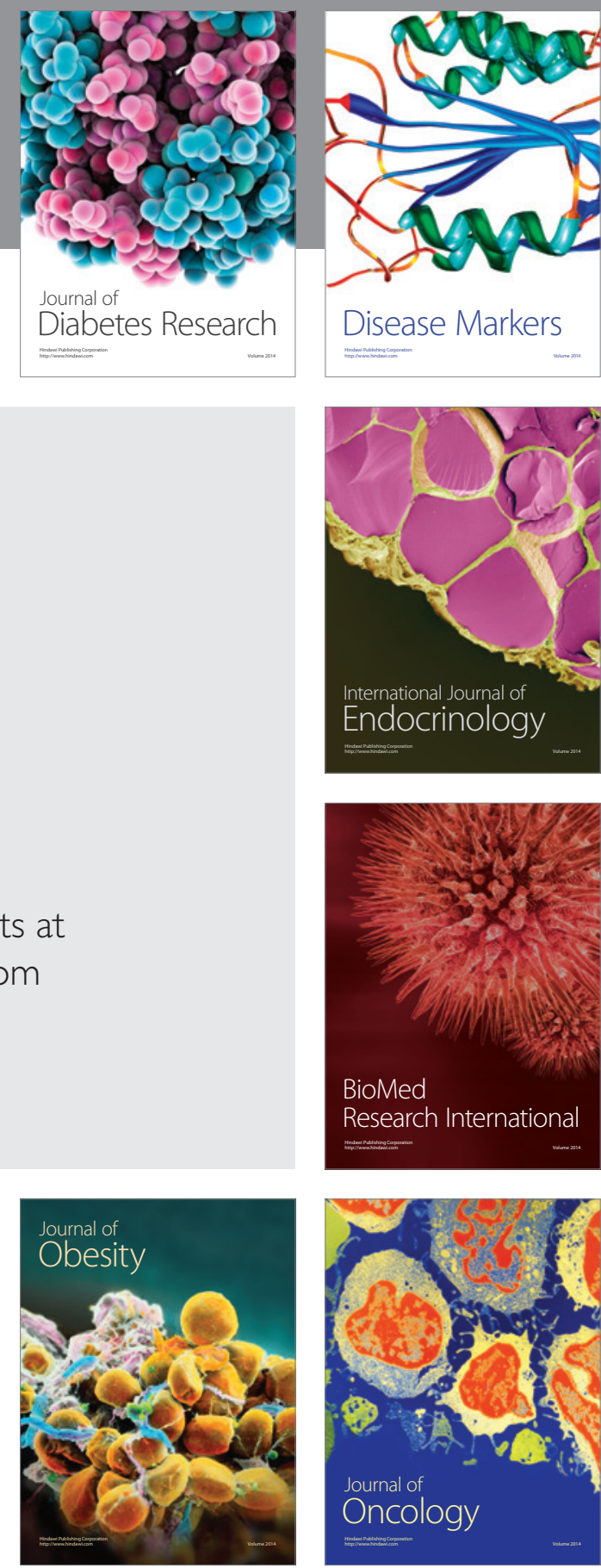

Disease Markers
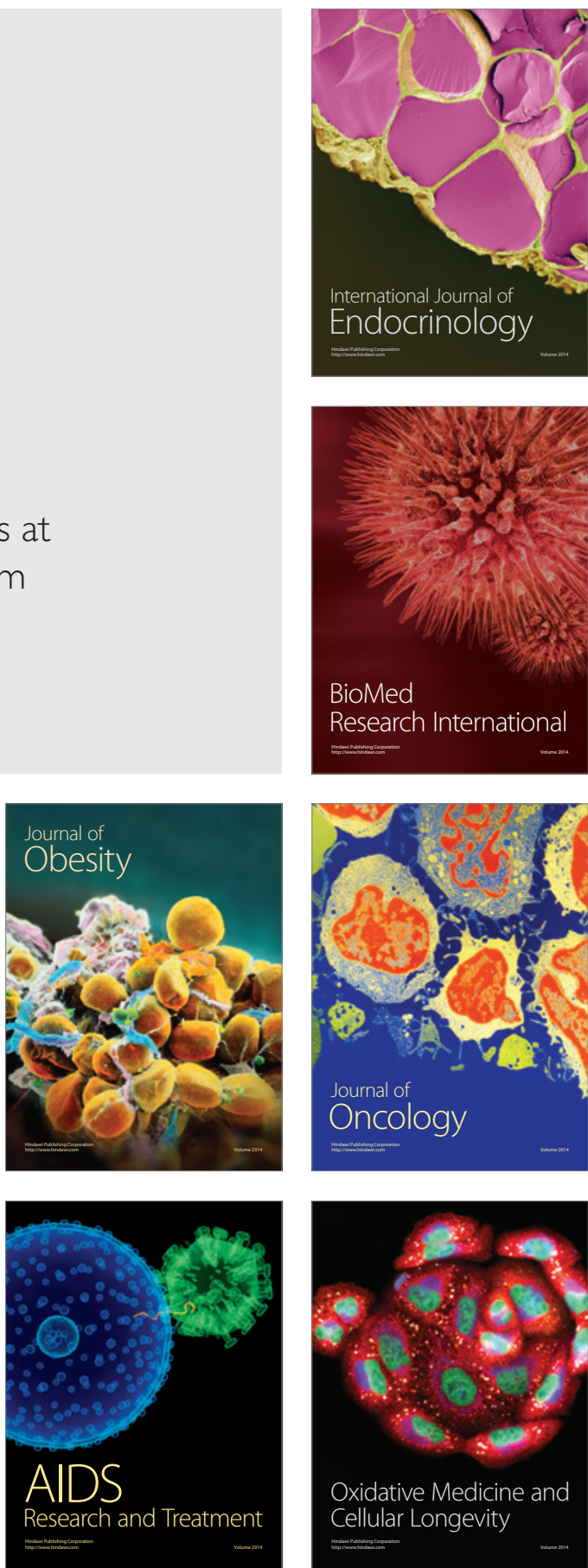